\title{
Maternal near miss and mortality in a rural referral hospital in northern Tanzania: a cross-sectional study
}

Ellen JT Nelissen ${ }^{1,2^{*}}$, Estomih Mduma ${ }^{1}$, Hege L Ersdal ${ }^{1,3}$, Bjørg Evjen-Olsen ${ }^{4,5}$, Jos JM van Roosmalen ${ }^{6,7}$ and Jelle Stekelenburg ${ }^{8}$

\begin{abstract}
Background: Maternal morbidity and mortality in sub-Saharan Africa remains high despite global efforts to reduce it. In order to lower maternal morbidity and mortality in the immediate term, reduction of delay in the provision of quality obstetric care is of prime importance. The aim of this study is to assess the occurrence of severe maternal morbidity and mortality in a rural referral hospital in Tanzania as proposed by the WHO near miss approach and to assess implementation levels of key evidence-based interventions in women experiencing severe maternal morbidity and mortality.
\end{abstract}

Methods: A prospective cross-sectional study was performed from November 2009 until November 2011 in a rural referral hospital in Tanzania. All maternal near misses and maternal deaths were included. As not all WHO near miss criteria were applicable, a modification was used to identify cases. Data were collected from medical records using a structured data abstraction form. Descriptive frequencies were calculated for demographic and clinical variables, outcome indicators, underlying causes, and process indicators.

Results: In the two-year period there were 216 maternal near misses and 32 maternal deaths. The hospital-based maternal mortality ratio was 350 maternal deaths per 100,000 live births (95\% Cl 243-488). The maternal near miss incidence ratio was 23.6 per 1,000 live births, with an overall case fatality rate of $12.9 \%$. Oxytocin for prevention of postpartum haemorrhage was used in 96 of 201 women and oxytocin for treatment of postpartum haemorrhage was used in 38 of 66 women. Furthermore, eclampsia was treated with magnesium sulphate in $87 \%$ of all cases. Seventy-four women underwent caesarean section, of which 25 women did not receive prophylactic antibiotics. Twenty-eight of 30 women who were admitted with sepsis received parenteral antibiotics. The majority of the cases with uterine rupture (62\%) occurred in the hospital.

Conclusion: Maternal morbidity and mortality remain challenging problems in a rural referral hospital in Tanzania. Key evidence-based interventions are not implemented in women with severe maternal morbidity and mortality. Progress can be made through up scaling the use of evidence-based interventions, such as the use of oxytocin for prevention and treatment of postpartum haemorrhage.

Keywords: Maternal near miss, Severe acute maternal morbidity, Maternal mortality, Quality of obstetric care, WHO near miss approach, WHO near miss criteria

\footnotetext{
* Correspondence: ejtnelissen@gmail.com

${ }^{1}$ Haydom Lutheran Hospital, 9000 Haydom, Manyara, Tanzania

${ }^{2}$ Athena Institute, Faculty of Earth and Life Sciences, VU University

Amsterdam, de Boelelaan 1085, 1081 HV Amsterdam, Netherlands

Full list of author information is available at the end of the article
} 


\section{Background}

Despite global efforts through the Safe Motherhood Initiative and the declaration of the Millennium Development Goals (MDG), there is only a slight decline in maternal mortality in sub-Saharan Africa [1,2]. Although Tanzania shows progress towards improving maternal health, as is shown by a $47 \%$ decline in maternal mortality ratio (MMR) between 1990 and 2010 (from an estimated MMR of 870 per 100,000 live births in 1990 to a MMR of 460 per 100,000 live births in 2010), maternal mortality remains high [3]. The aim of MDG 5 is to reduce maternal mortality by $75 \%$ between 1990 and 2015 [4]. Although the numbers of maternal deaths (MD) around the globe are high, absolute numbers in hospitals are low. Therefore, including cases of women who almost die, but survive pregnancy-related complications, is progressively being used to study the quality of obstetric care [5-8]. These near miss cases represent most of the characteristics of maternal deaths [9]. Combined, they create larger numbers and may lead to more rapid and precise reporting. Since identification criteria for near miss cases are not uniform and studies thus not comparable [6,7], WHO developed a new definition of maternal near miss (MNM) and identification criteria for maternal near miss cases in 2009 [10]. This resulted in the "WHO near miss approach for maternal health" in 2011 [11].

Currently, in Tanzania half of the deliveries take place in health facilities and 51\% of all deliveries are assisted by a skilled provider [12]. Unfortunately skilled birth attendance does not necessarily equal good quality of care [13]. Many evidence-based effective approaches are available to prevent and treat obstetric emergencies, but their implementation lags behind $[14,15]$. Human resources are scarce and policymakers encourage scaling up facility deliveries. Increased utilization of skilled birth attendants at facilities with insufficient human resources may further threaten quality of care. Negative perception of the quality of care by the community inhibits the decision to deliver in a facility $[16,17]$ and so a vicious circle is created. When scaling up skilled birth attendance, there is a need for a well-functioning health system with sufficient resources and a good infrastructure. Both, quantity of human resources and quality of care should be addressed [13,18].

In order to reduce maternal mortality and severe acute maternal morbidity, it is imperative not only to increase the numbers of skilled personnel, but also to improve access to health institutions and to reduce the delay in providing quality obstetric care within institutions. Quality of obstetric care can be measured using outcome indicators, such as case fatality rate (CFR), and process indicators, such as the use of oxytocin after delivery to prevent postpartum haemorrhage $[11,19]$.
This study aims to assess the occurrence of severe maternal morbidity and mortality in a referral hospital in rural Tanzania as proposed by the WHO near-miss approach and to assess implementation levels of key evidence-based interventions in women experiencing severe maternal morbidity and mortality.

\section{Methods}

This study is part of an intervention study. Data that were collected serve as baseline for an intervention with simulation-based training in routine delivery care, prevention and treatment of postpartum haemorrhage $(\mathrm{PPH})$. This was a prospective cross-sectional study, conducted from November 2009 until November 2011 in Haydom Lutheran Hospital (HLH), a referral hospital in rural Northern Tanzania. HLH is a 400-bed hospital owned by the Mbulu Diocese of the Evangelical Lutheran Church in Tanzania. The hospital provides free reproductive and child health services, comprehensive emergency obstetric care, including ambulance and radio service. Furthermore there is an Intensive Care Unit (ICU) with 24-hours medical supervision and mechanical ventilation. Annually there are around 5000 deliveries. Extrapolating from the 2002 census, the immediate catchment area was covering a population of approximately 327,000 in 2010 [20]. The greater reference area covered a population of approximately 2,200,000 people [20].

\section{Outcome measures}

The primary outcome measures were the total number of $\mathrm{MD}, \mathrm{MNM}$ and live births (LB) in the hospital during the study period. Subsequently, outcome indicators were calculated such as the number of women with lifethreatening conditions. As proposed by the WHO approach, overall near miss indicators were calculated such as the severe maternal outcome ratio, the maternal near miss incidence ratio, the maternal near-miss mortality ratio, and the case fatality rate. Hospital access indicators were calculated such as the number of women with life-threatening conditions at arrival, the proportion of these women among all women with life-threatening conditions, the proportion of these women coming from other hospitals, and the women with life-threatening conditions at arrival mortality index. Lastly, intra hospital care indicators were calculated: the number of maternal near misses and deaths who developed these conditions in the hospital and the intra hospital mortality index.

Implementation levels of evidence-based interventions were measured, such as use of oxytocin for prevention and treatment of $\mathrm{PPH}$, use of magnesium sulphate for treatment of eclampsia, use of prophylactic antibiotics during caesarean section, use of parenteral antibiotics 
for treatment of sepsis, and the proportion of women with uterine rupture that occurred in the hospital.

\section{Inclusion criteria}

All maternal deaths and maternal near misses that were admitted to HLH were prospectively included in the study during the above-mentioned period. A maternal death is defined as: the death of a woman while pregnant or within 42 days of termination of pregnancy from any cause. A maternal near miss is defined as: a woman who nearly died but survived a complication that occurred during pregnancy, childbirth or within 42 days of termination of pregnancy [10]. The intention was to use the WHO near miss criteria for the identification of maternal near misses. However, not all WHO criteria were applicable, and the identification criteria were adapted to the local situation in HLH as is described elsewhere [21]. Table 1 shows an overview of the WHO near miss criteria and the Haydom modification.

\section{Data collection}

Cases were identified on a daily basis by either the principal investigator (EN) or by one of the two trained research assistants (nurse-midwives). This was achieved through daily participation in the morning report and daily visits to the maternity ward, ICU and the internal medicine ward. When the inclusion criteria were met, a structured data abstraction form was filled out by the principal investigator or a research assistant. Data were obtained from the patient record. The facility medical staff was questioned in case of doubt or missing information. General information and obstetric details were collected. For the hospital access and intra hospital care indicators, the following information was registered: health status on arrival, maternal death within 24 hours, and referral status. For each case, one underlying cause was identified that started the cascade that led to maternal morbidity or mortality [22]. For example, a primipara was admitted with obstructed labour and had a caesarean section. After caesarean section she developed sepsis. The underlying cause that started the cascade that led to maternal morbidity or mortality was obstructed labour. This table shows one diagnosis per woman (mutually exclusive, totally inclusive). For the process indicators, information on preventive measures (measuring of vital signs, use of oxytocin or other uterotonics for prevention of $\mathrm{PPH}$, use of prophylactic antibiotics during caesarean section) were registered, as well as the use of interventions (use of oxytocin or other uterotonics as treatment for PPH, IV-infusion, blood products, hysterectomy, magnesium sulphate or other anticonvulsant in case of eclampsia, parenteral therapeutic antibiotics, and laparotomy for uterine rupture).

\section{Quality assessment of the data}

The completed data abstraction forms were checked by a second person on missing data or discrepancies. If needed, a copy of the hospital file was checked to validate the recordings. All MD cases were reviewed by a selection of the authors (EN, BEO, JVR and JS), as well as a random selection of the MNM cases and those cases, which were difficult to classify into an underlying cause. Observation bias was addressed by means of auditing all MD cases and a random sample of the MNM cases by four authors (EN, BEO, JVR, JS) until consensus was reached. All data were double entered and cross-checked in Epidata [23].

\section{Statistical analysis}

Data analysis consisted of frequencies of demographic and clinical variables and underlying causes. Demographic variables were cross-tabulated for maternal outcome at discharge, and compared using chi-square test for categorical variables and t-test for continuous variables. Outcome indicators were calculated using the total number of live births during the study period and the total number of MNM and MD in that same period. Descriptive frequencies were calculated for underlying causes and process indicators. All results are reported as number (n) and frequency (\%). Analysis was performed using SPSS Statistics, version 20 (SPSS Inc. Chicago, Illinois).

\section{Ethical approval}

Ethical approval was obtained from the Tanzanian National Institute for Medical Research (NIMR) (reference NIMR/HQ/R.8a/Vol.IX/1247), the Tanzania Commission for Science and Technology (COSTECH) (reference 2012-56-NA-2011-201), and from the VU university medical center (VUmc), the Netherlands (reference 2011/389). Data were collected and extracted from patient records without identification of the subject. Data abstraction forms were filled in after discharge or death and therefore study inclusion did not have effect on the treatment. Considering these precautions, individually obtained informed consent was not required.

\section{Results}

In the two-year study period 248 women with lifethreatening conditions were included at Haydom Lutheran Hospital: 216 maternal near miss cases and 32 maternal deaths. In the study period 9471 deliveries and 9136 live births occurred at HLH.

Demographic and clinical characteristics are shown in Table 2. There were no statistical differences in characteristics between the group of maternal near miss and the group of maternal deaths. The Datoga tribe, who are pastoralists, experienced relatively more deaths than 
Table 1 WHO near miss criteria adapted to the local context of HLH (reproduced from Nelissen et al.) WHO near miss criteria Haydom near miss criteria

\section{Clinical criteria}

\begin{tabular}{|c|c|}
\hline Acute cyanosis & Acute cyanosis \\
\hline Gasping & Gasping \\
\hline Respiratory rate $>40$ or $<6 / \mathrm{min}$ & Respiratory rate $>40$ or $<6 / \mathrm{min}$ \\
\hline Shock & Shock $^{a}$ \\
\hline Oliguria non responsive to fluids or diuretics & Oliguria non responsive to fluids or diuretics ${ }^{b}$ \\
\hline Failure to form clots & Failure to form clots ${ }^{c}$ \\
\hline Loss of consciousness lasting $>12 \mathrm{~h}$ & Loss of consciousness lasting $>12 \mathrm{~h}^{\mathrm{d}}$ \\
\hline Cardiac arrest & Cardiac arrest $^{e}$ \\
\hline Stroke & Stroke $^{f}$ \\
\hline Uncontrollable fit/total paralysis & Uncontrollable fit/total paralysis ${ }^{g}$ \\
\hline Jaundice in the presence of pre-eclampsia & Jaundice in the presence of pre-eclampsia ${ }^{h}$ \\
\hline \multicolumn{2}{|l|}{ Laboratory-based criteria } \\
\hline Oxygen saturation $<90 \%$ for $\geq 60$ minutes & Oxygen saturation $<90 \%$ for $\geq 60$ minutes \\
\hline \multicolumn{2}{|l|}{$\mathrm{PaO} 2 / \mathrm{FiO} 2<200$ mmHg } \\
\hline \multicolumn{2}{|l|}{ Creatinine $\geq 300 \mu \mathrm{mol} / \mathrm{l}$ or $\geq 3.5 \mathrm{mg} / \mathrm{dL}$} \\
\hline \multicolumn{2}{|l|}{ Bilirubin $>100 \mu \mathrm{mol} / \mathrm{l}$ or $>6.0 \mathrm{mg} / \mathrm{dL}$} \\
\hline \multicolumn{2}{|l|}{$\mathrm{pH}<7.1$} \\
\hline \multicolumn{2}{|l|}{ Lactate $>5 \mathrm{mEq} / \mathrm{mL}$} \\
\hline Acute thrombocytopenia $(<50,000$ platelets $/ \mathrm{ml})$ & Acute thrombocytopenia $(<50,000$ platelets/ml) \\
\hline \multicolumn{2}{|l|}{ Loss of consciousness and ketoacids in urine } \\
\hline \multicolumn{2}{|l|}{ Management-based criteria } \\
\hline & Admission to intensive care unit \\
\hline \multicolumn{2}{|l|}{ Use of continuous vasoactive drugs } \\
\hline Hysterectomy following infection or haemorrhage & Hysterectomy following infection or haemorrhage \\
\hline Transfusion of $\geq 5$ units of blood & Transfusion of $\geq 1$ unit of blood \\
\hline Intubation and ventilation for $\geq 60$ minutes not related to anaesthesia & Intubation and ventilation for $\geq 60$ minutes not related to anaesthesia \\
\hline \multicolumn{2}{|l|}{ Dialysis for acute renal failure } \\
\hline Cardio-pulmonary resuscitation & Cardio-pulmonary resuscitation \\
\hline \multicolumn{2}{|l|}{ Severe maternal complications } \\
\hline & Eclampsia $^{i}$ \\
\hline & Sepsis or severe systemic infection ${ }^{j}$ \\
\hline & Uterine rupture $^{k}$ \\
\hline
\end{tabular}

Applicability of the WHO near miss criteria in a low resource setting. PLoS One 2013, 8:e61248.

a: Shock is defined as a persistent severe hypotension, defined as a systolic blood pressure $<90 \mathrm{mmHg}$ for $60 \mathrm{~min}$ with a pulse rate of $\geq 120 / \mathrm{min}$ despite aggressive fluid replacement (> $2 \mathrm{~L}$ ).

b: Oliguria is defined as an urinary output $<30 \mathrm{ml} / \mathrm{hour}$ for 4 hours or $<400 \mathrm{ml} / 24 \mathrm{hr}$.

c: Failure to form clots is defined as the absence of clotting from the IV site after 7-10 minutes.

$\mathrm{d}$ : Unconsciousness/coma lasting $>12$ hours is defined as a profound alteration of mental state that involves complete or near-complete lack of responsiveness to external stimuli or Glasgow Coma Scale $<10$.

e: Cardiac arrest is defined as loss of consciousness and absence of pulse or heart beat.

$\mathrm{f}$ : Stroke is defined as a neurological deficit of cerebrovascular cause that persists $\geq 24$ hours, or is interrupted by death within 24 hours.

$\mathrm{g}$ : Uncontrollable fit is a condition in which the brain is in state of continuous seizure.

$\mathrm{h}$ : Pre-eclampsia: the presence of hypertension associated with proteinuria. Hypertension is defined as a blood pressure $\geq 140 \mathrm{mmHg}$ (systolic) or $\geq 90 \mathrm{mmHg}$ (diastolic). Proteinuria is defined as excretion of $\geq 300 \mathrm{mg}$ protein/24 hr or $300 \mathrm{mg}$ protein/litre urine or $\geq 1+$ on a dipstick.

i: Eclampsia is defined as the presence of hypertension associated with proteinuria and fits. Hypertension is defined as a blood pressure $\geq 140 \mathrm{mmHg}$ (systolic) or $\geq 90 \mathrm{mmHg}$ (diastolic). Proteinuria is defined as excretion of $\geq 300 \mathrm{mg}$ protein $/ 24 \mathrm{hr}$ or $300 \mathrm{mg}$ protein/litre urine or $\geq 1+$ on a dipstick.

j: Sepsis is defined as a clinical sign of infection and 3 of the following: temp $>38^{\circ} \mathrm{C}$ or $<36^{\circ} \mathrm{C}$, respiration rate $>20 / \mathrm{min}$, pulse rate $>90 / \mathrm{min}$, WBC $>12$.

$\mathrm{k}$ : Uterine rupture is defined as the complete rupture of a uterus (including peritoneum) with (partial) extrusion of the fetus during labour.

near misses, but this did not reach statistical significance. Almost $20 \%$ of all near misses $(n=40)$ and $22 \%$ of all maternal deaths $(n=7)$ had a gestational age of less than 24 weeks on admission. For $42 \%$ of all near misses $(\mathrm{n}=91)$ gestational age was 36 weeks or more, compared to $25 \%$ of all maternal deaths $(n=8)$. The most common 
Table 2 Demographic and clinical characteristics

\begin{tabular}{|c|c|c|c|}
\hline & $\begin{array}{c}\text { MNM } \\
n=216\end{array}$ & $\begin{array}{c}M D \\
n=32\end{array}$ & p-value \\
\hline Age mean (SD) in years & $28.1(6.3)$ & $\begin{array}{l}29.4 \\
(6.1)\end{array}$ & $p=0.266^{*}$ \\
\hline$<20$ years $\mathrm{n}(\%)$ & $20(9 \%)$ & $3(9 \%)$ & \\
\hline $20-35$ years $n(\%)$ & $\begin{array}{l}150 \\
(69 \%)\end{array}$ & $20(63 \%)$ & \\
\hline$\geq 35$ years $n(\%)$ & $46(21 \%)$ & $9(28 \%)$ & \\
\hline $\begin{array}{l}\text { Marital status } \\
\text { n (\%) }\end{array}$ & & & $p=0.341^{* * *}$ \\
\hline Single/separated/divorced & $21(10 \%)$ & $5(16 \%)$ & \\
\hline Married/cohabiting & $\begin{array}{l}188 \\
(87 \%)\end{array}$ & $25(78 \%)$ & \\
\hline Unknown & $7(3 \%)$ & $2(6 \%)$ & \\
\hline Tribe n (\%) & & & $p=0.477^{* *}$ \\
\hline Datoga & $31(14 \%)$ & $7(22 \%)$ & \\
\hline Iraqw & $\begin{array}{c}114 \\
(53 \%)\end{array}$ & $14(44 \%)$ & \\
\hline Other & $61(28 \%)$ & $9(28 \%)$ & \\
\hline Unknown & $10(5 \%)$ & $2(6 \%)$ & \\
\hline Parity n (\%) & & & $p=0.742^{* *}$ \\
\hline 0 & $47(22 \%)$ & $5(16 \%)$ & \\
\hline $1-2$ & $52(24 \%)$ & $7(22 \%)$ & \\
\hline$\geq 3$ & $\begin{array}{c}100 \\
(46 \%)\end{array}$ & $16(50 \%)$ & \\
\hline Unknown & $17(8 \%)$ & $4(13 \%)$ & \\
\hline Previous caesarean section n (\%) & & & $p=0.307^{* * *}$ \\
\hline No & $\begin{array}{l}145 \\
(67 \%)\end{array}$ & $24(75 \%)$ & \\
\hline Yes & $41(19 \%)$ & $3(9 \%)$ & \\
\hline Unknown & $30(14 \%)$ & $5(16 \%)$ & \\
\hline Gestational age $\mathrm{n}(\%)$ & & & $p=0.106^{* *}$ \\
\hline$<24$ weeks & $40(19 \%)$ & $7(22 \%)$ & \\
\hline 24-36 weeks & $40(19 \%)$ & $10(31 \%)$ & \\
\hline$\geq 36$ weeks & $91(42 \%)$ & $8(25 \%)$ & \\
\hline Unknown & $45(21 \%)$ & $7(22 \%)$ & \\
\hline Mode of delivery $n(\%)$ & & & $* * * *$ \\
\hline Vaginal delivery & $80(37 \%)$ & $15(47 \%)$ & \\
\hline Caesarean section & $69(32 \%)$ & $5(16 \%)$ & \\
\hline Laparotomy for uterine rupture & $16(7 \%)$ & $1(3 \%)$ & \\
\hline Abortion/curettage & $22(10 \%)$ & $2(6 \%)$ & \\
\hline $\begin{array}{l}\text { Laparotomy for ectopic } \\
\text { pregnancy }\end{array}$ & $19(9 \%)$ & - & \\
\hline Undelivered & $9(4 \%)$ & $9(28 \%)$ & \\
\hline Unknown & $1(1 \%)$ & - & \\
\hline Fetal presentation n (\%) & & & $* * * *$ \\
\hline Cephalic & $\begin{array}{c}110 \\
(51 \%)\end{array}$ & $15(47 \%)$ & \\
\hline Breech & $10(5 \%)$ & $1(3 \%)$ & \\
\hline Other & $9(4 \%)$ & $1(3 \%)$ & \\
\hline
\end{tabular}

Table 2 Demographic and clinical characteristics (Continued)

\begin{tabular}{lcl}
\hline Abortion & $40(19 \%)$ & $4(13 \%)$ \\
\hline Unknown & $47(22 \%)$ & $11(34 \%)$ \\
\hline${ }^{*}$ Independent sample t-test; ${ }^{* *}$ Chi-square test; ${ }^{* *}$ Fisher exact test (2-sided);
\end{tabular}

**** $\mathrm{p}$-value could not be calculated due to too few cases.

mode of delivery was vaginal delivery: $37 \%$ MNM ( $\mathrm{n}=$ $80)$ and $47 \%$ MD $(n=15)$. This was followed by caesarean section: $32 \%$ MNM $(n=69)$ and 16\% MD $(n=5)$. There were no assisted vaginal deliveries (e.g. forceps/ vacuum extraction). Almost $30 \%$ of all women who died $(\mathrm{n}=9)$ died undelivered.

As is shown in Table 3, there were 27.1 MNM and MD per 1,000 live births; this is also referred to as the severe maternal outcome ratio. For every maternal death there were 6.8 near miss cases. Hospital-based MMR was 350 per 100,000 live births (95\% CI 243-488), with an overall CFR of $12.9 \%$. Nearly $70 \%$ of all women with life-threatening conditions were ill on arrival and 20.9\% were referred from another hospital. Death within 24 hours occurred in $7.6 \%(n=13)$ of the women that were ill on arrival. Seventy-six women (30.6\%) developed a life-threatening condition in the hospital, and four (5.3\%) of these women died as a result of this condition.

Table 4 demonstrates the underlying causes for both groups (MNM and MD) and the associated CFR per underlying cause. The major cause of morbidity and mortality is postpartum haemorrhage, accounting for $27 \%$ of all underlying causes, followed by abortion related complications (17\%), obstructed labour (12\%), ante partum haemorrhage $(11 \%)$, and hypertensive disorders (9\%). Women who got severely ill from an unknown cause had the highest risk of dying with a CFR of $80 \%$, followed by cancer (67\%) and non-obstetric infectious diseases (63\%).

Process indicators that reflect the use of evidencebased interventions are presented in Table 5. Two hundred one women gave birth in the hospital, and 96 $(48 \%)$ received oxytocin as part of active management of third stage of labour (AMTSL). Sixty-six women were diagnosed with severe PPH. Forty-one of these women (62\%) delivered in the hospital and 25 women (38\%) delivered at home or on their way to the hospital. Twentyeight women (42\%) who were diagnosed with severe $\mathrm{PPH}$ had received oxytocin routinely after delivery. The number of women that received oxytocin as treatment was higher: 38 (58\%). Ten women received ergometrine and/or misoprostol in addition to oxytocin. Fifty-four women $(82 \%)$ received intravenous volume replacement, whereas 61 women (92\%) received blood transfusion. In four women (6\%) hysterectomy was performed. 
Table 3 Outcome indicators

\begin{tabular}{|c|c|}
\hline Outcomes & $\begin{array}{l}\text { Near-miss } \\
\text { indicators }\end{array}$ \\
\hline All live births in the hospital (n) & 9136 \\
\hline \multicolumn{2}{|l|}{ Severe maternal outcomes } \\
\hline Maternal deaths $(n)$ & 32 \\
\hline Maternal near miss cases $(n)$ & 216 \\
\hline Women with life-threatening conditions (WLTC) (n) & 248 \\
\hline \multicolumn{2}{|l|}{ Overall near miss indicators } \\
\hline $\begin{array}{l}\text { Severe maternal outcome ratio (SMOR) (per 1,000 } \\
\text { live births) }\end{array}$ & 27.1 \\
\hline $\begin{array}{l}\text { Maternal near miss incidence ratio (per 1,000 live } \\
\text { births) }\end{array}$ & 23.6 \\
\hline Maternal near miss mortality ratio & 6.75 \\
\hline Case fatality rate $(\%)$ & $12.9 \%$ \\
\hline \multicolumn{2}{|l|}{ Hospital access indicators } \\
\hline WLTC at hospital arrival (n) & 172 \\
\hline Proportion WLTC at arrival among all WLTC (\%) & $69.4 \%$ \\
\hline $\begin{array}{l}\text { Proportion of WLTC at arrival coming from other } \\
\text { hospitals (\%) }\end{array}$ & $20.9 \%$ \\
\hline WLTC at arrival mortality index (\%) & $7.6 \%$ \\
\hline \multicolumn{2}{|l|}{ Intra hospital care indicators } \\
\hline Intra hospital WLTC cases ( $\mathrm{n}$ ) & 76 \\
\hline Intra hospital WLTC rate (per 1,000 live births) & 8.32 \\
\hline Intra hospital mortality index (\%) & $5.3 \%$ \\
\hline
\end{tabular}

Live birth (LB): the complete expulsion or extraction from its mother of a product of conception, irrespective of the duration of the pregnancy, which, after such separation, breathes or shows any other evidence of life. Each product of such a birth is considered live born.

Women with life-threatening conditions (WLTC): the sum of maternal near miss and maternal deaths. (WLTC $=$ MNM + MD).

Severe Maternal Outcome Ratio (SMOR): the number of women with lifethreatening conditions per 1,000 live births. [SMOR $=(M N M+M D) / L B]$. MNM incidence ratio: the number of maternal near miss cases per 1,000 live births. [MNM IR = MNM/LB].

Maternal near miss : mortality ratio: the proportion between maternal near miss cases and maternal deaths. [MNM : $1 \mathrm{MD}$ ].

Case fatality Rate: the number of maternal deaths divided by the number of women with life-threatening conditions, expressed as a

percentage. [CFR $=$ MD/(MNM + MD)].

WLTC at hospital arrival: the number of women with life-threatening conditions who are ill on arrival.

Proportion WLTC at arrival among all WLTC: the number of WLTC who are ill on arrival divided by the total number of WLTC.

Proportion of WLTC at arrival coming from other hospitals: the number of WLTC who are ill on arrival and coming from another hospital divided by the total number of WLTC at arrival.

WLTC at arrival mortality index: the maternal deaths within 24 hours after arrival (MD24) divided by the number of women with life-threatening conditions who were ill on arrival, expressed as percentage. [WLTC at arrival $\mathrm{Ml}=\mathrm{MD} 24 /(\mathrm{WLTC}$ at arrival)].

Intra hospital WLTC cases: the number of women with life-threatening conditions who developed these life-threatening conditions in the hospital. Intra hospital WLTC rate (per 1000 live births): the number of women with lifethreatening conditions who developed these life-threatening conditions in the hospital per 1000 live births.

Intra hospital mortality index: the number of maternal deaths who were not ill on arrival, divided by the number of women with life-threatening conditions who were not ill on arrival, expressed as percentage.

Fifteen women were diagnosed with eclampsia, of which 13 women (87\%) received magnesium sulphate. Ten women received diazepam, of which nine also
Table 4 Underlying causes of maternal morbidity and mortality (mutually exclusive, totally inclusive)

\begin{tabular}{|c|c|c|c|}
\hline & & n (\%) & CFR $(\%)^{*}$ \\
\hline \multirow[t]{4}{*}{ Indirect } & Anaemia in pregnancy & $19(8 \%)$ & $0 \%$ \\
\hline & Infectious disease & $11(4 \%)$ & $63 \%$ \\
\hline & Cardiac disease & $4(2 \%)$ & $50 \%$ \\
\hline & Cerebral disease & $2(1 \%)$ & $50 \%$ \\
\hline \multirow[t]{9}{*}{ Direct } & Postpartum haemorrhage & $67(27 \%)$ & $9 \%$ \\
\hline & Abortion related complication & $43(17 \%)$ & $7 \%$ \\
\hline & Ante partum haemorrhage & $\begin{array}{l}26 \\
(11 \%)\end{array}$ & $8 \%$ \\
\hline & Hypertensive disorders & $21(9 \%)$ & $10 \%$ \\
\hline & Obstructed labour with uterine rupture & $15(6 \%)$ & $13 \%$ \\
\hline & $\begin{array}{l}\text { Obstructed labour without uterine } \\
\text { rupture }\end{array}$ & $15(6 \%)$ & $7 \%$ \\
\hline & $\begin{array}{l}\text { Uterine scar rupture without } \\
\text { obstructed labour }\end{array}$ & $6(2 \%)$ & $0 \%$ \\
\hline & Puerperal sepsis & $9(4 \%)$ & $0 \%$ \\
\hline & Anaesthesia related complication & $1(0.4 \%)$ & $0 \%$ \\
\hline \multirow{2}{*}{$\begin{array}{l}\text { Co- } \\
\text { incidental }\end{array}$} & Cancer & $3(1 \%)$ & $67 \%$ \\
\hline & Trauma & $1(0.4 \%)$ & $0 \%$ \\
\hline Unknown & Unknown/undetermined cause & $5(2 \%)$ & $80 \%$ \\
\hline
\end{tabular}

* CFR: case fatality rate, calculated as MD/(MD + MNM).

received magnesium sulphate, indicating that 93\% women received anticonvulsants.

Seventy-four women underwent a caesarean section, of which $49(66 \%)$ received prophylactic antibiotics. Twelve women (16\%) developed sepsis after caesarean section and one woman died. Six of the women (50\%) who developed sepsis received prophylactic antibiotics.

Twenty-eight of the women (93\%) that were diagnosed with sepsis received parenteral therapeutic antibiotics.

The last process indicator regarding uterine rupture revealed that the majority of the ruptures occurred inhospital (62\%). All women underwent laparotomy, and one woman died. CFR for uterine rupture was $5 \%$.

Of all women with life-threatening conditions, 227 women (92\%) had physical examination on arrival. One hundred seventy-two women were ill on arrival and 133 (77\%) had blood pressure measured, 124 (72\%) had pulse rate taken and of 126 women (73\%) temperature was noted. Saturation was measured in only 10 women (6\%). Full blood count was taken in 155 (90\%) of the women that were ill on arrival.

\section{Discussion}

This article presents the results of a prospective crosssectional study describing the quality of obstetric care in a rural referral hospital in Tanzania using the modified WHO near-miss criteria. It is one of the few studies 
Table 5 Process indicators among women with severe maternal morbidity and mortality

\begin{tabular}{ll}
\hline 1. Prevention of PPH & n (\%) \\
\hline Target population: women who delivered in hospital & $201(100 \%)$ \\
\hline Oxytocin use for AMTSL & $96(48 \%)$ \\
\hline Other uterotonic use for AMTSL & $2(1 \%)$ \\
\hline All uterotonic use for AMTSL & $96(48 \%)$ \\
\hline 2. Treatment of PPH & $66(100 \%)$ \\
\hline Target population: women with PPH & $41(62 \%)$ \\
\hline \multicolumn{1}{c}{ Delivery in hospital } & $25(38 \%)$ \\
\hline \multicolumn{1}{|c|}{ Delivery out hospital } & $28(42 \%)$ \\
\hline Oxytocin use (routine) & - \\
\hline Other uterotonic use (routine) & $28(42 \%)$ \\
\hline All uterotonic use (routine) & $38(58 \%)$ \\
\hline Oxytocin use (treatment) & $10(15 \%)$ \\
\hline Other uterotonic use (treatment) & $39(59 \%)$ \\
\hline All uterotonic use (treatment) & $54(82 \%)$ \\
\hline IV-infusion & $61(92 \%)$ \\
\hline Blood products & $4(6 \%)$ \\
\hline Hysterectomy & $9 \%$ \\
\hline CFR &
\end{tabular}

3. Treatment of eclampsia

\begin{tabular}{ll}
\hline Target population: women with eclampsia & $15(100 \%)$ \\
\hline Magnesium sulphate use & $13(87 \%)$ \\
\hline Other anticonvulsant use & $10(67 \%)$ \\
\hline Any anticonvulsant use & $14(93 \%)$ \\
\hline CFR & $7 \%$
\end{tabular}

\section{Prevention of caesarean section related infection}

\begin{tabular}{ll}
\hline Target population: women with caesarean section & 74 (100\%) \\
\hline Prophylactic antibiotics & $49(66 \%)$ \\
\hline
\end{tabular}

\section{Treatment of sepsis}

\begin{tabular}{ll}
\hline Target population: women with sepsis & $30(100 \%)$ \\
\hline Parenteral therapeutic antibiotics & $28(93 \%)$ \\
\hline CFR & $27 \%$ \\
\hline 6. Uterine rupture & $21(100 \%)$ \\
\hline Target population: women with uterine rupture & $13(62 \%)$ \\
\hline Occurred in hospital & $8(38 \%)$ \\
\hline Occurred out hospital & $21(100 \%)$ \\
\hline Laparotomy & $5 \%$
\end{tabular}

PPH: postpartum haemorrhage, this is defined as the loss of $500 \mathrm{ml}$ of blood or more; AMTSL: active management of third stage of labour; CFR: case fatality rate, calculated as $\mathrm{MD} /(\mathrm{MD}+\mathrm{MNM})$

describing maternal morbidity and mortality in Tanzania [24-28].

MMR in this hospital was 350 maternal deaths per 100,000 live births (95\% CI 243-488). Country level MMR is around 460 per 100,000 live births [3,12]. Previous studies in the area revealed MMRs of $362(95 \%$
CI 269-456) and 444 (95\% CI 371-517) per 100,000 live births in 1995 and 1996 for household and antenatal clinic surveys, respectively [25]. It is difficult to compare the hospital-based calculated MMR with a population-based MMR because not all women deliver nor die inside the hospital. Most likely hospital-based MMR is an overestimation because $48 \%$ of the Tanzanian women deliver at home and $85 \%$ of all maternal deaths are identified in-hospital [12,29]. Having this in mind, our estimation of MMR is relatively low compared to the national MMR, as is underlined by previous research from the area. This may be explained by the free service of the hospital for delivery care and the wellestablished transport services. Furthermore, although the hospital did not have medical specialists, there were frequent long-term expatriate specialists and visiting specialists from nearby referral hospitals.

A recently published systematic review was conducted that included all studies on maternal near miss from 2004 until 2010 [7]. It revealed that inclusion criteria used to identify maternal near miss varied widely and therefore could not easily be compared. The findings showed MNM prevalence rates that varied from $0.04 \%$ to $14.98 \%$ depending on the different inclusion criteria that were used. These results were further subdivided into regions and the findings from 14 studies from Africa showed that there is a near-miss prevalence rate ranging from $0.05 \%$ to $14.98 \%$. The nearmiss prevalence in HLH of $2.48 \%$ lies within the lower range of the studies from Africa that were included in this analysis. From the fourteen studies that were analyzed in the sub-group Africa, seven studies used management specific criteria (emergency hysterectomy or ICU admission) and had relatively low near-miss rates, as they did not include all near misses that were admitted to the hospital.

Although maternal mortality and morbidity ratios are relatively low compared to the rest of the country, quality of care can still be improved as is shown by the process indicators. The improvement lies in the up scaling of evidence-based interventions. As suggested by Campbell et al. "we should get on with what works" in order to reduce maternal mortality [28,30]. Implementation of evidence-based interventions such as oxytocin use immediately after delivery for prevention of postpartum haemorrhage can be improved in HLH. Only $48 \%$ of the women received routine oxytocin immediately after delivery, while WHO recommends this for all women who give birth [31]. WHO also recommends the use of oxytocin for the treatment of $\mathrm{PPH}$ [32], however in HLH it was used in $58 \%$ of the cases with severe $\mathrm{PPH}$. PPH was the leading cause of maternal morbidity and the second cause for maternal mortality in HLH with a CFR of $9 \%$. Confronting hospital 
staff with these low numbers of oxytocin use reveals several possible reasons. Not recording the use of oxytocin in the hospital file is the main reason, followed by lack of protocols, shortage of staff, and high staff turnover resulting in less experienced health care workers. Supply of oxytocin to the ward is not considered a constraint. Although there were no official guidelines in HLH available, local policy was to give $10 \mathrm{IU}$ of oxytocin immediately after birth and it was also the first drug of choice for the treatment of PPH. Use of oxytocin for prevention and treatment of $\mathrm{PPH}$ should be scaled up to $100 \%$ coverage.

Caesarean section seems to be one of the most important risk factors for postpartum maternal infection [33,34]. Prophylactic antibiotics reduces endometritis and decreases wound infections [35]. At HLH it was used only in $66 \%$ of all women who underwent caesarean section. Twelve women (16\%) developed sepsis after caesarean section and one woman died. Of the women who developed sepsis, six women (50\%) received prophylactic antibiotics. Sepsis accounted for $4 \%$ of all maternal morbidities in HLH and the number of women with sepsis after caesarean section may be reduced by this simple precaution. Prophylactic use of antibiotics during caesarean section should be scaled up to $100 \%$.

Use of magnesium sulphate in case of severe preeclampsia is recommended as it reduces the risk of eclampsia with $50 \%$ and might reduce maternal mortality $[36,37]$. Eclampsia is not very common at HLH regarding the incidence of $1.6 \%$ per 1,000 deliveries, but it does account for $6 \%$ of all women with life-threatening conditions. Case fatality rate for eclampsia was $7 \%$. Nearly $90 \%$ of all eclamptic women received magnesium sulphate. Hypertensive disorders are typically detected during antenatal care (ANC) visits. ANC coverage in Tanzania is relatively good: $96 \%$ of the pregnant women visit the ANC once and $43 \%$ visit the ANC four times or more. Most women visit the antenatal clinic 2-3 times. In Manyara region $68.2 \%$ of the women have blood pressure measured during ANC visits [12]. ANC visits are a good opportunity to detect hypertensive disorders, and blood pressure should be measured on every occasion.

The majority of uterine ruptures occurred in-hospital , which reflects serious substandard care. Obstructed labour was prevalent in $12 \%$ of all maternal morbidities and mortalities. Uterine rupture is one of the medical emergencies with high maternal and perinatal mortality as is reflected by the maternal CFR of $5 \%[38,39]$. Other hospital-based studies from low-income countries have reported case fatality rates ranging from 1 to $13 \%$ [40]. Audit can be used to reduce the incidence of uterine rupture as is shown in Malawi [41]. Some of the recommendations that were implemented in Malawi after several audit sessions were training of healthcare workers in order to reduce delay in diagnosing obstructed labour and delay in treatment and implementation of local guidelines for augmentation of labour. Increased supervision should lead to improved documentation and labour management.

There were several limitations to this study. First, it is a single-centre and hospital-based study and therefore findings may not reflect the situation in other settings or the population in general. Second, information is gathered from hospital files, therefore data quality depends on the quality of record keeping. We have tried to remedy this by means of conducting prospective datacollection. Third, for the collection of maternal near miss cases we have used modified WHO near miss criteria, as the original WHO near miss criteria were not applicable in the local context. This has to be taken into account when interpreting the results from this study, as there have been more near miss cases collected with the modified criteria, then would have been with the original criteria [21].

\section{Conclusion}

The present study shows that, although the hospital-based maternal mortality ratio is quite low compared to the country-level maternal mortality ratio, maternal morbidity and mortality remain challenging problems in a rural referral hospital in Tanzania. The WHO near miss criteria used for identification of near miss cases could not all be applied in this low-resource setting. Therefore the inclusion criteria were modified for use in Haydom. Results show that there is a gap between actual and optimal use of evidence-based interventions in women experiencing severe maternal morbidity and mortality. Improvement in the quality of obstetric care can be made through up scaling the use of evidence-based interventions.

\section{Abbreviations}

AMTSL: Active management of third stage of labour; CFR: Case fatality rate; $\mathrm{Cl}$ : Confidence interval; HLH: Haydom lutheran hospital; ICU: Intensive care unit; MD: Maternal death; MDG: Millennium development goal;

MMR: Maternal mortality ratio; MNM: Maternal near miss; PPH: Postpartum haemorrhage; SD: Standard deviation; WLTC: Women with life-threatening conditions.

\section{Competing interests}

EN received a stipend from the Laerdal Foundation for Acute Medicine, Stavanger, Norway. The sponsor of the study had no role in study design, data collection, data analysis, data interpretation, or writing of the report.

\section{Authors' contributions}

EN was involved in the conception and design of the study, carried out the analysis and wrote the first draft of the paper. EM, HLE, BEO, JVR and JS assisted with the conception and design of the study and contributed to the writing of the paper. BEO, JS and JVR also participated in the classification of diagnoses for all MNM and MD cases. All authors read and approved the final draft of the paper. 


\section{Acknowledgements}

The authors wish to thank the management, research assistants Sarah Lyanga and Victoria Bayda, and staff of Haydom Lutheran Hospital for their efforts to make this project happen. This gratitude extends to Erling Svensen, who supervised the project, Øystein Evjen Olsen, who was involved in the early phase of designing the study and Sven Gudmund Hinderaker, who assisted with the analysis of the data and the fine-tuning of the draft. Furthermore we would like to thank the Laerdal Foundation for Acute Medicine, Stavanger, Norway for their financial support. Finally we would like to thank Jacqueline Broerse, professor at the VU University Amsterdam, for her valuable input during the writing of the paper.

\section{Author details}

${ }^{1}$ Haydom Lutheran Hospital, 9000 Haydom, Manyara, Tanzania. ${ }^{2}$ Athena Institute, Faculty of Earth and Life Sciences, VU University Amsterdam, de Boelelaan 1085, 1081 HV Amsterdam, Netherlands. ${ }^{3}$ Stavanger Acute Medicine Foundation for Education and Research (SAFER), Stavanger University Hospital, POB 8100, 4068 Stavanger, Norway. ${ }^{4}$ Centre for International Health, University of Bergen, Årstadveien 21, N-5009 Bergen, Norway. ${ }^{5}$ Department of Obstetrics and Gynaecology, Sørlandet Hospital, Engvald Hansens vei 6, 4400 Flekkefjord, Norway. ${ }^{6}$ Department of Obstetrics, Leiden University Medical Centre, Albinusdreef 2, 2333 Leiden, ZA, Netherlands. ${ }^{7}$ Department of Medical Humanities, EMGO Institute for Health and Care Research, VU University Medical Centre, Van der Boechorststraat 7, 1081 BT Amsterdam, Netherlands. ${ }^{8}$ Department of Obstetrics \& Gynaecology, Leeuwarden Medical Centre, Henri Dunantweg 2, 8934 AD Leeuwarden, Netherlands.

Received: 11 November 2012 Accepted: 28 June 2013 Published: 4 July 2013

\section{References}

1. Hogan MC, Foreman KJ, Naghavi M, Ahn SY, Wang M, Makela SM, Lopez AD, Lozano R, Murray CJ: Maternal mortality for 181 countries, 19802008: a systematic analysis of progress towards Millennium Development Goal 5. Lancet 2010, 375:1609-1623.

2. Lozano R, Wang H, Foreman KJ, Rajaratnam JK, Naghavi M, Marcus JR, Dwyer-Lindgren L, Lofgren KT, Phillips D, Atkinson C, et al: Progress towards Millennium Development Goals 4 and 5 on maternal and child mortality: an updated systematic analysis. Lancet 2011, 378:1139-1165.

3. World Health Organization, UNICEF, UNFPA, The World Bank: Trends in Maternal Mortality: 1990 to 2010. WHO, UNICEF, UNFPA and The World Bank estimates. World Health Organization; 2012.

4. United Nations General Assembly: United Nations Millennium Declaration. A/RES/55/2. 192000. UN General Assembly, 55th session, agenda item 60(b).

5. Pattinson R: Near misses: a useful adjunct to maternal death enquiries Br Med Bull 2003, 67:231-243.

6. Say L, Pattinson RC, Gülmezoglu AM: WHO systematic review of maternal morbidity and mortality: the prevalence of severe acute maternal morbidity (near miss). Reprod Heal 2004, 1:3.

7. Tuncalp O, Hindin MJ, Souza JP, Chou D, Say L: The prevalence of maternal near miss: a systematic review. BJOG 2012, 119:653-661.

8. Kaye DK, Kakaire O, Osinde MO: Systematic review of the magnitude and case fatality ratio for severe maternal morbidity in sub-Saharan Africa between 1995 and 2010. BMC Pregnancy Childbirth 2011, 11:65.

9. Pattinson RC, Buchmann E, Mantel G, Schoon M, Rees H: Can enquiries into severe acute maternal morbidity act as a surrogate for maternal death enquiries? BJOG 2003, 110:889-893.

10. Say L, Souza JP, Pattinson RC: Maternal near miss-towards a standard tool for monitoring quality of maternal health care. Best Pract Res Clin Obstet Gynaecol 2009, 23:287-296.

11. World Health Organization: Evaluating the quality of care for severe pregnancy complications. The WHO near-miss approach for maternal health. World Health Organization; 2011.

12. National Bureau of Statistics (NBS) [Tanzania] and ICF Macro: Tanzania Demographic and Health Survey 2010 Final Report. Dar es Salaam, Tanzania: NBS and ICF Macro; 2011

13. Koblinsky M, Matthews Z, Hussein J, Mavalankar D, Mridha MK, Anwar I, Achadi E, Adjei S, Padmanabhan P, Marchal B, et al: Going to scale with professional skilled care. Lancet 2006, 368:1377-1386.
14. Graham WJ: Criterion-based clinical audit in obstetrics: bridging the quality gap? Best Pract Res Clin Obstet Gynaecol 2009, 23:375-388.

15. Freedman LP, Graham WJ, Brazier E, Smith JM, Ensor T, Fauveau V, Themmen E, Currie S, Agarwal K: Practical lessons from global safe motherhood initiatives: time for a new focus on implementation. Lancet 2007, 370:1383-1391.

16. Kruk ME, Rockers PC, Mbaruku G, Paczkowski MM, Galea S: Community and health system factors associated with facility delivery in rural Tanzania: a multilevel analysis. Health Policy 2010, 97:209-216.

17. Gabrysch S, Campbell OM: Still too far to walk: literature review of the determinants of delivery service use. BMC Pregnancy Childbirth 2009, 9:34

18. Mathai $\mathrm{M}$ : To ensure maternal mortality is reduced, quality of care needs to be monitored and improved alongside increasing skilled delivery coverage rates. BJOG 2011, 118(Suppl 2):12-14.

19. Janakiraman V, Ecker J: Quality in obstetric care: measuring what matters. Obstet Gynecol 2010, 116:728-732

20. The United Republic of Tanzania: 2002. Population and housing census- village and street statistics, age and sex distribution, Manyara Region. In Edited by Central Census Office NBoS, President's Offices, Planning and Privatisation. Dar es Salaam; 2005.

21. Nelissen E, Mduma E, Broerse J, Ersdal H, Evjen-Olsen B, Van Roosmalen J, Stekelenburg J: Applicability of the WHO Maternal Near Miss Criteria in a Low-Resource Setting. PLoS One 2013, 8:e61248.

22. World Health Organization: International statistical classification of diseases and related health problems. ; 2011. 10th revision, edition 2010. World Health Organization.

23. Lauritsen J, Bruus M: EpiData. A comprehensive tool for validated entry and documentation of data. Odense, Denmark: The EpiData Association; 2003.

24. Nyamtema AS, De Jong AB, Urassa DP, Van Roosmalen J: Using audit to enhance quality of maternity care in resource limited countries: lessons learnt from rural Tanzania. BMC Pregnancy Childbirth 2011, 11:94.

25. Olsen BE, Hinderaker SG, Kazaura M, Lie RT, Bergsjo P, Gasheka P, Kvale G: Estimates of maternal mortality by the sisterhood method in rural nothern Tanzania: a household sample and an antenatal clinic sample. BJOG 2000, 107:1290-1297.

26. Mbaruku G, Van Roosmalen J, Kimondo I, Bilango F, Bergstrom S: Perinatal audit using the 3-delays model in western Tanzania. Int J Gynaecol Obstet 2009, 106:85-88

27. Francesconi P, Pisani V: Estimating maternal mortality by the Sisterhood method in Iringa District. Tanzania. Trop Doct 2001, 31:220.

28. Sorensen BL, Elsass P, Nielsen BB, Massawe S, Nyakina J, Rasch V: Substandard emergency obstetric care - a confidential enquiry into maternal deaths at a regional hospital in Tanzania. Trop Med Int Health 2010, 15:894-900.

29. Olsen BE, Hinderaker SG, Lie RT, Bergsjo P, Gasheka P, Kvale G: Maternal mortality in northern rural Tanzania: assessing the completeness of various information sources. Acta Obstet Gynecol Scand 2002, 81:301-307.

30. Campbell OM, Graham WJ: Strategies for reducing maternal mortality: getting on with what works. Lancet 2006, 368:1284-1299.

31. World Health Organization: WHO recommendations for the prevention of postpartum haemmorhage. World Health Organization; 2007.

32. World Health Organization: WHO guidelines for the management of postpartum haemorrhage and retained placenta. World Health Organization; 2009.

33. Gibbs RS: Clinical risk factors for puerperal infection. Obstet Gynecol 1980, 55:178S-184S.

34. Declercq E, Barger M, Cabral HJ, Evans SR, Kotelchuck M, Simon C, Weiss J, Heffner LJ: Maternal outcomes associated with planned primary cesarean births compared with planned vaginal births. Obstet Gynecol 2007, 109:669-677.

35. Smaill FM, Gyte GM: Antibiotic prophylaxis versus no prophylaxis for preventing infection after cesarean section. Cochrane Database Syst Rev 2010. CD007482.

36. Duley L, Gulmezoglu AM, Henderson-Smart DJ, Chou D: Magnesium sulphate and other anticonvulsants for women with pre-eclampsia. Cochrane Database Syst Rev 2010. CD000025.

37. Altman D, Carroli G, Duley L, Farrell B, Moodley J, Neilson J, Smith D: Do women with pre-eclampsia, and their babies, benefit from magnesium sulphate? The Magpie Trial: a randomised placebo-controlled trial. Lancet 2002, 359:1877-1890. 
38. Ali AA, Adam I: Maternal and perinatal outcomes of uterine rupture in the Kassala Hospital, east Sudan: 2006-2009. J Obstet Gynaecol 2011, 31:48-49.

39. Aboyeji AP, ljaiya MD, Yahaya UR: Ruptured uterus: a study of 100 consecutive cases in llorin, Nigeria. J Obstet Gynaecol Res 2001, 27:341-348.

40. Hofmeyr GJ: Obstructed labor: using better technologies to reduce mortality. Int J Gynaecol Obstet 2004, 85(Suppl 1):S62-72.

41. van den Akker T, Mwagomba B, Irlam J, Van Roosmalen J: Using audits to reduce the incidence of uterine rupture in a Malawian district hospital. Int J Gynaecol Obstet 2009, 107:289-294.

doi:10.1186/1471-2393-13-141

Cite this article as: Nelissen et al:: Maternal near miss and mortality in a rural referral hospital in northern Tanzania: a cross-sectional study. BMC Pregnancy and Childbirth 2013 13:141.

\section{Submit your next manuscript to BioMed Central and take full advantage of:}

- Convenient online submission

- Thorough peer review

- No space constraints or color figure charges

- Immediate publication on acceptance

- Inclusion in PubMed, CAS, Scopus and Google Scholar

- Research which is freely available for redistribution 